\title{
LE IDEE SCIENTIFICHE COME PROTESI DELLA MENTE
}

\author{
PAOLO MAZZARELLO (*)
}

SUNTO. - Nella storia intellettuale italiana è difficile trovare una mente, come quella di Carlo Cattaneo, che sia stata altrettanto in grado di rifrangersi in mille rivoli, frantumarsi in mille percorsi, suddividersi in mille suggestioni, sempre però unificata da una spinta ispirativa unitaria di passione per la razionalità e la scienza. Economia, storia, psicologia, geografia, politica, etica, chimica, tecnologia - in una lunga catena di interessi, una sorta di onnivora e insaziabile bulimia conoscitiva - furono le cifre di un intellettuale appassionato delle cose del mondo, sempre pronto a lasciarsi sedurre dalle innovazioni e dalle idee scientifiche in grado di influire sulla società. In bilico fra illuminismo - alla cui tradizione era legato - e positivismo, ma in realtà non precisamente inquadrabile nella tassonomia intellettuale ottocentesca del nostro paese, Cattaneo è un unicum che a distanza di quasi centocinquant'anni dalla sua scomparsa mantiene una forte vitalità come simbolo di valori di cui si sente forte il bisogno. La «fiducia nel progresso attraverso la ragione» e dunque la lotta contro ogni forma di «settarismo» e di populismo, la convinta difesa delle libertà civili e politiche, la democrazia e conseguentemente «il controllo del potere dal basso», l'idea di autogoverno e dell'autonomia di scelta di una comunità, la logica della pratica scientifica come modello del miglioramento razionale del vivere sociale. Alla base vi era la passione per il progresso scientifico e tecnologico, coefficiente dell'avanzamento umano, e il contenuto educativo e dunque profondamente etico della divulgazione delle conoscenze prodotte dall'innovazione e dall'esplorazione del mondo, di quella che gli inglesi chiamerebbero ora public understanding of scien$c e$. La riflessione sulla natura della scienza e l'indagine relativa alle modalità del suo svilupparsi in una società, sono al centro del volume di Carlo Cattaneo Psicologia delle menti associate. Introdotta da Carlo Lacaita e ricomposta da Barbara Boneschi, l'opera deriva soprattutto dal mosaico di conferenze, legate da un tessuto unitario, tenute dall'autore all'Istituto Lombardo fra il 1859 e il 1866. Questa serie di letture che Cattaneo avrebbe poi voluto far confluire in un'opera organica, furono un momento importante della sua elaborazione intellettuale, uno spazio di indagine che andò al cuore dei suoi interessi perché collocato al centro unitario da cui si sprigionava la sua attività, lo spirito scientifico che tingeva tutta la sua vorace tensione conoscitiva. In questo commento

(*) Istituto Lombardo Accademia di Scienze e Lettere, Milano. Università degli Studi di Pavia, Italia. E-mail: paolo.mazzarello@unipv.it 
verrà data particolare enfasi a due particolari aspetti della psicologia scientifica di Cattaneo. Da un lato l'idea del sapere scientifico come impresa collettiva, dunque come sintesi collaborativa di più menti associate. Dall'altro la concezione della scienza come dispositivo cognitivo che rende possibile l'amplificazione della possibilità di esplorazione e percezione del mondo.

$* * *$

ABSTRACT. - The reflection on the nature of science and the investigation concerning the modalities of its development in a society, are at the center of the volume of Carlo Cattaneo Psicologia delle menti associate (Psychology of the associated minds). Introduced by Carlo Lacaita and organized by Barbara Boneschi, the work derives above all from the mosaic of lectures, linked by a unitary vision, held by the author at the Istituto Lombardo between 1859 and 1866. This series of readings were an important moment of his intellectual elaboration, a space of investigation that went to the heart of his cultural interests, the scientific knowledge that involved all his voracious cognitive tension. In this comment, particular emphasis will be given to two particular aspects of Cattaneo's scientific psychology. On the one hand, the idea of scientific knowledge as a collective enterprise, therefore as a collaborative synthesis of several associated minds; on the other hand, the concept of science as a cognitive device that makes it possible to amplify the possibility of exploration and perception of the world.

Nella storia intellettuale italiana è difficile trovare una mente, come quella di Carlo Cattaneo, che sia stata altrettanto in grado di rifrangersi in mille rivoli e frantumarsi in molteplici percorsi, sempre però unificata da una spinta unitaria di passione per la razionalità e la scienza.

Economia, storia, psicologia, geografia, politica, etica, chimica, tecnologia - in una lunga catena di interessi, una sorta di onnivora e insaziabile bulimia conoscitiva - furono le cifre di un intellettuale appassionato delle cose del mondo, sempre pronto a lasciarsi sedurre dalle innovazioni e dalle idee scientifiche in grado di influire sulla società. Come meglio non poteva dirsi, il sottotitolo del Politecnico portava la specificazione Repertorio mensile di studj applicati alla prosperità e coltura sociale. Nella premessa al primo volume lo scopo del periodico esprimeva il desiderio di diffondere fra i cittadini «la più pronta cognizione di quella parte di vero che dalle aride regioni della Scienza può facilmente condursi a fecondare il campo della Pratica, e crescere sussidio e conforto alla prosperità comune ed alla convivenza civile» ${ }^{1}$.

1 Il Politecnico. Repertorio mensile di studj applicati alla prosperità e coltura sociale, 1, anno 1, semestre primo, 3; cfr. C.G. Lacaita, L'organizzatore di cultura e "Il Politecnico", in I volti di Carlo Cattaneo. Un grande italiano del Risorgimento, a cura di F. Della Peruta, C.G. Lacaita e F. Mazzocca, Skira, 2001, 63-71. 
Dunque non solo il sapere per il sapere, ma la conoscenza come strumento militante di educazione e trasformazione della realtà sociale.

In bilico fra illuminismo - alla cui tradizione era legato - e positivismo, ma in realtà non precisamente inquadrabile nella tassonomia intellettuale ottocentesca del nostro paese, Cattaneo è un unicum intellettuale che a distanza di quasi centocinquant'anni dalla sua scomparsa mantiene una forte vitalità come simbolo di valori di cui si sente forte il bisogno. La «fiducia nel progresso attraverso la ragione» e dunque la lotta contro ogni forma di «settarismo» e di populismo, la convinta difesa delle libertà civili e politiche, la democrazia e conseguentemente «il controllo del potere dal basso», l'idea di autogoverno e dell'autonomia di scelta di una comunità ${ }^{2}$, la logica della pratica scientifica come modello del miglioramento razionale del vivere sociale. Alla base vi era la passione per il progresso scientifico e tecnologico - coefficiente dell'avanzamento umano - e il contenuto educativo e dunque profondamente etico della divulgazione delle conoscenze prodotte dall'innovazione e dall'esplorazione del mondo, di quella che gli autori anglosassoni chiamerebbero ora public understanding of science.

$\mathrm{Al}$ centro di questo atteggiamento intellettuale e culturale stava dunque la grande considerazione che Cattaneo aveva per la scienza e la sua applicazione, cioè la tecnologia. La riflessione sulla sua natura e l'indagine relativa alle modalità del suo svilupparsi in una società, sono al centro dell'opera che presentiamo oggi. Introdotta sapientemente da Carlo Lacaita e ricomposta in maniera unitaria da Barbara Boneschi, la Psicologia delle menti associate deriva soprattutto dal mosaico di letture interconnesse tenute da Carlo Cattaneo fra il 1859 e il 1866 all'Istituto Lombardo, l'accademia che - come ha scritto Mariachiara Fugazza - fu «il punto di riferimento più importante che avesse a Milano» in quegli anni ${ }^{3}$. Questa serie di conferenze che avrebbe poi voluto far confluire in un'opera organica, furono un momento importante della sua elaborazione intellettuale, uno spazio di indagine che andò al cuore dei suoi interessi perché collocato al centro unitario da cui si sprigionava la sua

2 N. Bobbio, Carlo Cattaneo nel primo centenario della morte, in «Paragone/Letterature», 1973, n. 278; cfr. F. Focher, Libertà e teoria dell'ordine politico. Machiavelli, Guicciardini e altri studi, FrancoAngeli, 2000, 128.

3 M. Fugazza, Carlo Cattaneo. Scienza e società 1850-1868, FrancoAngeli, 1989, 43. 
attività, lo spirito scientifico che tingeva tutta la sua vorace tensione conoscitiva.

Vi è una domanda in quest'opera, un punto di partenza fondamentale di psicologia sociale e antropologia culturale, ben espresso già dalla prefazione aggiunta dalla curatrice, una domanda inquieta che si pone Carlo Cattaneo: «Come avvenne che al cospetto del medesimo universo, uomini che la scienza suppone in origine dotati delle identiche facultà di percepire e d'idealizzare, siano pervenuti a condizioni mentali sì enormemente disparate, che fra loro non v'è più adito a un pensiero commune?» ${ }^{4}$ Se, come scrive Cattaneo, la «più alta formula della morale è quella che insegna all'uomo di considerare ogni suo simile come un altro sé stesso» ${ }^{5}$, da che cosa deriva l'abissale differenza per quanto riguarda la conoscenza e la capacità di incidere nel mondo fra un popolo rimasto all'età della pietra e un europeo del diciannovesimo secolo? «Perché» - si domanda Cattaneo - «Polifemo non ha visto anch'egli i circoli d'Archimede?» pur influenzati entrambi dallo stesso mare, ed esposti alla luce dello stesso cielo e non differenti «per fatto di natura, e di psicologia primitiva» ${ }^{6}$ ?

Contrariamente a quello che pensava Montesquieu - dice Cattaneo - non sono i climi che hanno «somma influenza [...] nella genesi delle civiltà e perciò anche delle dottrine». L'esperienza della storia insegna che vi sono popoli vissuti in condizioni ambientali e di vita materiale diametralmente opposti ad altri e tuttavia in grado di produrre straordinarie innovazioni: l'India le cifre decimali, gli arabi «il concetto o almeno il nome dell'algebra e della chimica» e gli scozzesi il logaritmo. Newton, continua, «visse nel più nebuloso dei climi»e Linneo «visse tra le nevi della Svezia». Dunque non sono di certo i climi e le condizioni materiali della vita che a queste conseguono a determinare il genio delle nazioni. Non solo, vi sono anche popoli, come i greci, che vissero nello stesso clima lungo le epoche, produttori di grandi civiltà, in certi periodi, caduti poi nella più profonda «impotenza mentale», o viceversa le stirpi «sì lungamente barbare degli Scandinavi, dei

4 C. Cattaneo, Psicologia delle menti associate, in Memorie dell'Istituto Lombardo - Accademia di Scienze e Lettere, Classe di Scienze Morali e Storiche, XLV, 89304, part. 93.

5 Ibid., 102.

6 Ibid., 91-92. 
Germani, degli Slavi, dei Magiari [...] iniziarsi nelle scienze tanto disprezzate dai loro padri» ${ }^{7}$.

Il problema del sapere diventa dunque subito un problema di psicologia sociale e con questa premessa si sviluppa l'indagine di Cattaneo, «sulle menti associate». Perché, sostiene l'autore, la psicologia è «lo studio delle facultà del pensiero», e la «più adulta e perfetta forma del nostro pensiero è la contemplazione scientifica - la contemplazione dell'ordine universale - dell'ordine nella natura e nell'umanità» ${ }^{8}$. A questo punto, dunque, la psicologia diventa la base dell'epistemologia, la via per giungere a stabilire la condizione stessa della conoscenza.

Per Cattaneo esistono delle forze fondamentali nel determinare l'origine delle idee, che paradossalmente sono estranee all'intelletto e tuttavia costituiscono «coefficienti potentissimi» del lavoro scientifico. In primo luogo l'istinto, «la facultà di compiere certi atti senza previa cognizione», cioè «l'azione senza idea». Proprietà psichica multipla che sgorga spontaneamente dalle profondità biologiche della psiche umana. Cattaneo considera in particolare l'istinto, che fa dell'uomo un essere sociale, presupposto della formazione di una società stabile, in secondo luogo l'istinto di imitazione «che se si eccettua qualche specie d'augelli e di scimie, è uno dei più caratteristici della specie umana; ed è di supremo momento non solo alla formazione della parola, ma in tutte le arti»? Gli istinti sono per Cattaneo il primum movens della disseminazione e dell'acquisizione delle idee scientifiche, il prerequisito della loro contaminazione associativa nello spazio, in senso trasversale fra individui contemporanei, e nel tempo attraverso le generazioni. Da qui, scrive ancora, «l'associazione delli avi ai posteri, dei maestri agli allievi, e la perpetua successione nell'immortale opera del sapere» ${ }^{10}$. Perché la scienza non è opera delle menti individuali, ma delle menti associate, espressa da una «commune intelligenza ${ }^{11} \mathrm{o}$, potremmo anche dire, di una mente collettiva che si sviluppa bene ed è favorita da un clima di libertà.

«Signori», esclama Cattaneo, «il lievito che fa fermentare le idee non si svolge in una mente sola; il genio si tien per mano alla catena de'

\footnotetext{
7 Ibid., 118-119.

8 Ibid., 115.

9 Ibid., 122.

10 Ibid.

11 Ibid., 182.
} 
suoi precursori. Perché si destino le idee, devono attuarsi i più generosi istinti, devono infervorarsi gli animi. La corrente del pensiero vuole una pila elettrica di più cuori e di più intelletti» ${ }^{12}$. Vi sono in Cattaneo delle espressioni che ricalcano, quasi anche a livello lessicale, moderne teorie della trasmissione culturale. Quando un esperto di botanica osserva analiticamente gli arbusti di una foresta cercando di riconoscerli e paragonarli mentalmente a quelli già classificati dalla scienza, «il suo pensiero», afferma lo studioso milanese, «non è un fenomeno primitivo e spontaneo, ma una produzione derivata e artificiale. Non è scaturito dalle sole facultà della sua mente; sono le menti di molti che pensano in lui> ${ }^{13}$. Dunque le idee sono dei "pacchetti" di conoscenze che saltano di mente in mente, dunque di cervello in cervello, esercitandovi un'influenza. Questo avviene perché l'istinto sociale pone in comunicazione gli individui e l'istinto di imitazione permette di acquisire dispositivi cognitivi di altri e di incorporarli in noi stessi. Sono in definitiva elementi di trasmissione culturale che si impiantano replicandosi all'interno di altre menti. A mio parere, facendo un po' di anacronismo provocatorio, più o meno quello che il genetista e filosofo della scienza inglese Richard Dawkins ha chiamato memi, unità replicative di trasmissione culturale o unità di imitazione. Vediamo provocatoriamente cosa scrive questo biologo inglese ne Il gene egoista: «Proprio come i geni si propagano nel pool genico saltando di corpo in corpo tramite spermatozoi o cellule uovo, così i memi si propagano nel pool memico saltando di cervello in cervello tramite un processo che, in senso lato, si può chiamare imitazione. Se uno scienziato sente o legge una buona idea, la passa ai suoi colleghi e studenti e la menziona nei suoi articoli e nelle sue conferenze. Se l'idea fa presa, si può dire che si propaga diffondendosi di cervello in cervello» ${ }^{14}$.

Ovviamente questo meccanismo di trasmissione del sapere è inibito al di fuori di un pabulum mentale collettivo che lo possa riconoscere, corrispondente in un certo senso al pool memetico di Dawkins, espresso dagli esseri sociali in grado di riconoscerlo e di acquisirlo imitandolo. Infatti, scrive Cattaneo, se a osservare gli stessi arbusti vi fossero dei selvaggi privi di competenza botanica «le loro facultà, indiffe-

\footnotetext{
12 Ibid., 123.

13 Ibid., 92.

14 R. Dawkins, Il gene egoista, Mondadori 1995, 201.
} 
renti, inerti», non potrebbero rispondere all'invito perché le «menti altrui non possono pensare in loro», cioè replicarsi in altre menti $1^{15}$.

Consideriamo ora un secondo fattore psichico, dopo l'istinto, che sta alla base della conoscenza: la sensazione. Subito Cattaneo enuncia un paradosso: «È vero che il selvaggio vive assorto nei sensi; è vero che l'esercizio assiduo e la dura necessità glieli rendono vigili e acuti. Ma s'egli avesse pure la vista dell'aquila e l'odorato del cane, sempre è vero che le sue sensazioni non hanno varietà» ${ }^{16}$. Sarebbero sensazioni limitate a superare le semplici difficoltà della vita, non adeguate a esplorare il mondo. Invece, scrive Cattaneo, la «sensazione scientifica abbraccia tutte le terre e tutti i mari; i vulcani e i ghiacciai, le pianure e i monti, gli arcipelaghi dispersi nell'Oceano e il deserto senz'aque». Una cognizione scientifica è un dispositivo cognitivo che permette di ampliare la conoscenza della realtà, uno strumento di esplorazione del mondo, in definitiva un ausilio protesico della mente. Così è ad esempio la chimica, ambito dell'attività umana che permette di rendere comprensibili fenomeni naturalmente inaccessibili ai sensi. Una conoscenza scientifica può trasformarsi anche in un dispositivo tecnico, come il telescopio e il microscopio, capace di svelare un mondo invisibile a occhio nudo. In senso lato questi apparati diventano quasi delle espansioni degli organi sensitivi, nel caso specifico la vista, cioè degli ausili protesici in grado di ampliare le possibilità dei sensi, senza sostituirsi a essi. Per estensione tutta la scienza strumentale ottocentesca può essere concepita come un gigantesco marchingegno in grado di far uscire dal cono d'ombra fenomeni ultrasensibili, rendendoli conoscibili alla mente umana. Scrive Cattaneo: «Ebbene, chi ci diede a scorta l'ago calamitato tra le nebbie dei mari, tra il polverio del deserto, tra i labirinti delle miniere, chi tese un telegrafo elettrico dall'uno all'altro declivio d'una montagna, dall'uno all'altro lido d'un mare ci fornì dunque un equivalente ad un nuovo senso, utile e reale quanto i sensi della vista e dell'udito. Nulla poi rileva all'effetto se sia un organo corporalmente inserito nel nostro encefalo, o se i nuovi fenomeni, rappresentandosi nello spazio colle vibrazioni d'un ago o d'un manubrio, si traducano nel senso della vista $\rangle^{17}$. Ausili mentali, e in taluni casi vere e proprie protesi cognitive percettive e motorie che

\footnotetext{
15 Cattaneo, Psicologia delle menti associate, cit. 93.

16 Ibid., 124.

17 Ibid., 126-127.
} 
la scienza mette a disposizione in soggetti, come i sordomuti o i ciechi nati che se non fossero «approdati alla vita in seno ad un consorzio già illuminato dalla filosofia non avrebbero potuto riparare colla sostituzione d'altro senso alle funzioni del senso mancante. Nel cieco nato che legge la parola colle dita, nel sordomuto che legge la parola sui moti delle labbra, una sensazione artificiale [...] supplisce alla incompleta sensazione naturale» ${ }^{18}$.

$\mathrm{Ma}$ in definitiva tutto il sapere e il metodo scientifico è anche un mezzo, uno strumento, un ausilio per esplorare il mondo. Le idee e le operazioni del sapere razionale sono dei dispositivi che si integrano nelle facoltà della mente per ampliare ulteriormente gli orizzonti della conoscenza; questi, a loro volta, si tramutano in rinnovati congegni intellettuali per ulteriori perlustrazioni del reale, evidentemente in un continuo e progressivo gioco di specchi, cioè di rimandi fra mezzi e fini.

L'integrazione delle menti individuali nelle menti associate, alle quali si devono le concezioni più generali, sono definibili sotto l'etichetta di sistema, cioè «una serie d'idee fra loro intimamente connesse per mezzo d'una idea principale o principio» che le coordina ${ }^{19}$. Vi sono tuttavia sistemi aperti, dinamici, che guardano al cambiamento, e sono quelli nei quali la scienza gioca un ruolo importante e altri chiusi, regressivi, quando «il despotismo [...] si pone in luogo della libertà»o quando «l'autorità [...] si pone in luogo della ragione» ${ }^{20}$, che diventano dunque «il sepolcro dell'intelligenza ${ }^{21}$. Talvolta un ordine d'idee che «dapprima fu progresso, divien poscia decadimento» ${ }^{22}$. Tanto maggiore è l'influenza del pensiero scientifico, tanto più un sistema si mantiene aperto potendo anche entrare in uno stato di gioventù perpetua «come appunto è ogni scienza esperimentale»» ${ }^{23}$. I suoi effetti ne segnano il destino: «Qualunque sia la potenza della tradizione religiosa, la vedrete venir meno inanzi all'autorità del nuovo principio che protende oltre i confini delle credenze» ${ }^{24}$. Di conseguen-

\footnotetext{
18 Ibid., 198-199.

19 Ibid., 141.

20 Ibid., 149.

21 Ibid., 152.

22 Ibid.

23 Ibid., 153.

24 Ibid., 102.
} 
za, sotto l'influenza della scienza, l'umanità tende al progresso. Ed è proprio l'Europa il motore avanzato di questa marcia, di questa «fausta prospettiva», la fucina dove «una forza espansiva preme e incalza i sistemi tradizionali» e proprio qui «l'inesauribilità del processo esperimentale, e la ragione di popoli, sciolta omai da ogni vincolo di tradizione, preparano al genere umano un'indefinita carriera e gli promettono una perpetua gioventù» $\gg^{25}$.

La scienza muta nel tempo e il progresso «continuo e indefinito» è la conseguenza della «successione» dei sistemi. Certi brani lascerebbero intravvedere scenari espressi quasi con il linguaggio kuhniano della sostituzione del paradigma, perlomeno nelle grandi concezioni scientifiche allo stato nascente, per esempio nello svilupparsi delle interpretazioni del moto planetario sulla base della meccanica celeste. Cattaneo scrive infatti: «La nuova idea non abbracciò dunque l'antica, ma la escluse; l'atto finale della mente non fu già un più complicato inganno, ma un disinganno, non un'addizione, additamentum, ma una sostituzione $\rangle^{26}$. Anche se, naturalmente, lo studioso milanese fu lontano dalla concezione kuhniana di mutamento della scienza.

Cattaneo aveva una mente che tendeva più alla conciliazione che allo scontro. Così come lo stato federale costituiva la sintesi di tendenze diverse e contrapposte che trovavano un terreno comune di unione delle diversità nell'unità, così le menti associate producevano anch'esse una sintesi collaborativa nell'ambito della scienza.

La sua stessa mente ne era un esempio, dispersa in mille rivoli, mille argomenti, mille interessi, tendeva tuttavia all'unità. Le continue forze centripete delle sue passioni intellettuali, la sua curiosità che lo spingeva in ogni direzione, ad assorbire criticamente ogni novità della scienza, della tecnica, del pensiero filosofico, la sua, potremmo dire, "intelligenza multipla" trovava poi sempre uno spazio unificato di condensazione della pluralità delle idee.

Era, per dirla con il linguaggio poetico di Isaiah Berlin ${ }^{27}$, derivato da un misterioso aforisma di Archiloco, "una volpe che voleva essere un riccio".

\footnotetext{
25 Ibid., 155.

26 Ibid., 98.

27 I. Berlin, Il riccio e la volpe, Adelphi, 1998.
} 